\title{
Combined strain and composition-induced effects in the metal-insulator transition of epitaxial $\mathrm{VO}_{2}$ films
}

\author{
V. Théry, ${ }^{1}$ A. Boulle, ${ }^{1}$ A. Crunteanu, ${ }^{2}$ and J. C. Orlianges ${ }^{2}$ \\ ${ }^{1}$ Sciences des Procédés Céramiques et Traitements de Surfaces, CNRS UMR 7315, \\ Centre Européen de la céramique, 12 rue Atlantis, 87068 Limoges Cedex, France \\ ${ }^{2}$ XLIM, UMR 7252 CNRS / Université de Limoges, \\ 123 Av. Albert Thomas, 87060 Limoges, France.
}

\begin{abstract}
The role of epitaxial strain, thermal strain, and bulk (strain-free) lattice parameter in the metalinsulator transition (MIT) and the structural phase transition (SPT) of $\mathrm{VO}_{2}$ is investigated for the case epitaxial films grown on (001)-oriented $\mathrm{TiO}_{2}$ substrates. Temperature-resolved X-ray reciprocal space mapping has been used to determine the absolute state of strain as well as the bulk lattice parameters of $\mathrm{VO}_{2}$ at $100^{\circ} \mathrm{C}$. For the thinnest film $(15 \mathrm{~nm})$, the state of strain is dominated by the film/substrate lattice mismatch yielding an in-plane tensile strain which, in turn, shifts both the MIT and the SPT towards lower temperatures. Conversely, for the thickest film (100 nm), the epitaxial strain is relaxed, so that state of strain is dominated by the $\mathrm{VO}_{2} / \mathrm{TiO}_{2}$ thermal expansion mismatch which is responsible for a compressive in-plane strain. In all cases a swelling of the strainfree $\mathrm{VO}_{2}$ unit-cell is observed which indicates the presence of interfacial oxygen vacancies and/or $\mathrm{Ti}$ diffusion into the $\mathrm{VO}_{2}$ films. The presence of oxygen vacancies stabilize the metallic rutile phase and counterbalances the action of thermal strain on the MIT and the SPT and degrade the electric properties for the thinnest film. For the thickest film the resistivity ratio is $6.4 \times 10^{4}$.
\end{abstract}

Oxide materials exhibiting a metal-insulator transition (MIT), such as vanadium dioxide $\left(\mathrm{VO}_{2}\right)$ are highly sensitive to elastic strain. In particular, the characteristics of the MIT can be profoundly modified by the application of strain as demonstrated by several studies in the last decades [1-16]. The role of strain in the MIT of $\mathrm{VO}_{2}$ is well explained within the generally accepted molecular orbital scheme, first proposed by Goodenough [17], and implying the hybridization of the $\mathrm{O} 2 \mathrm{p}$ orbitals with the V $3 \mathrm{~d}$ orbitals [18]. In the rutile (metallic) phase, the crystal field, associated with octahedral environment of the $\mathrm{V}$ ions, splits the $3 \mathrm{~d}$ level into a combination of a low energy $t_{2 g}$ and high energy $e_{g}^{\sigma}$ levels. The tetragonal distortion of the $\mathrm{VO}_{6}$ octahedron (with different apical and equatorial $\mathrm{V}-\mathrm{O}$ bond lengths), further splits the $t_{2 g}$ level into a single $a_{1 g}$ (also noted $d_{\|}$) orbital and a doubly degenerate $e_{g}^{\pi}$ orbital. The $d_{\|}$orbital is non-bonding with respect to the $\mathrm{O} 2 \mathrm{p}$ orbitals and is directed along the $c_{R}$ axis (where the subscript $\mathrm{R}$ stand for rutile), whereas the $e_{g}^{\pi, \sigma}$ orbitals hybridize with the $\mathrm{O} 2 \mathrm{p}$ orbitals to form low energy bonding $(\pi, \sigma)$ and high energy anti-bonding $\left(\pi^{*}, \sigma^{*}\right)$ levels. In the conducting state, the $d_{\|}$and $\pi^{*}$ orbitals are partly occupied by one electron per V atom and overlap at the Fermi level. When cooling down through the MIT temperature, $\mathrm{VO}_{2}$ evolves towards a monoclinic (M1) phase: the $\mathrm{V}$ ions undergo an anti-ferroelectric displacement parallel to $<110>_{R}$ which increases the p-d hybridization and up-shifts the $\pi^{*}$ level, whereas the formation of $\mathrm{V}-\mathrm{V}$ dimers along $[001]_{R}$ splits the $d_{\| \mid}$level into an occupied bonding $d_{\|}$ and empty anti-bonding $d_{\|}^{*}$ level, both effects yielding a $0.6 \mathrm{eV}$ gap $[17,18]$. The application of, say, compressive strain along $[001]_{R}$ reduces the p-d hybridization which in turns increases the overlap $d_{\|}-\pi^{*}$ overlap, hence stabilizing the metallic phase $[7,8]$.

Besides elastic strain, the stoichiometry of $\mathrm{VO}_{2}$ also plays a major role in the MIT. It has for instance been demonstrated that oxygen vacancies severely affects, or even completely suppresses, the MIT [3, 19-28]. The removal of oxygen ions locally reduce the $\mathrm{p}-\mathrm{d}$ hybridization thus resulting in the stabilization of the metallic phase. Furthermore, the oxygen vacancies act as donor levels hence increasing the electron density and up-shifting the Fermi level towards the valence band $[21-23,27]$.

Whereas the effect of strain and composition are well understood when considered separately, they are only rarely considered together $[29,30]$. Depending on the sign of the elastic strain, composition and strain may have cooperative or competing effects, that might lead to erroneous conclusions if both contributions are not correctly taken into account. This is especially relevant for transition metal oxides, exhibiting several possible oxidation states, in which unwanted composition fluctuations are easily introduced. Another widely overlooked effect is the differential film/substrate thermal expansion which, as shown below, can completely reverse strain geometry.

In the present Letter, we address the thicknessdependence of the metal-insulator transition (MIT) and the structural phase transition (SPT) of $\mathrm{VO}_{2}$ films epitaxially grown on (001)-oriented $\mathrm{TiO}_{2}$ substrates. It is demonstrated that a careful analysis of the state of strain of the $\mathrm{VO}_{2}$ films using X-ray diffraction (XRD) reciprocal space maps (RSMs) allows to disentangle the role of epitaxial strain, thermal strain, and composition of the films. Eventually, the behavior of both the MIT and the SPT, can be rationalized by taking into account these effects.

The $\mathrm{VO}_{2}$ films, with thickness 15,50 and $100 \mathrm{~nm}$, were grown by electron beam evaporation of a metallic vanadium target under oxygen atmosphere on (001)-oriented $\mathrm{TiO}_{2}$ substrates heated at $500^{\circ} \mathrm{C}$. The deposition rate was calibrated using ellipsometry measurements to de- 
termine the thickness of a series of test samples. The accuracy of the targeted film thickness is around 5\%. The films, irrespective of their thickness, do not develop any cracks or surface modification during the heating/colling cycles. Full details on the growth procedure can be found elsewhere [31, 32]. The MIT was characterized through resistivity measurements from $40^{\circ} \mathrm{C}$ to $100^{\circ} \mathrm{C}$ using the four-probes technique [31]. A Peltier element was used to control the heating / cooling of the samples while the temperature was monitored by a thermocouple attached near the films' surfaces. The structural properties of the films were investigated with temperature-resolved XRD measurements using a high-resolution Bruker "D8 discover" diffractometer $\left(\mathrm{Cu} \mathrm{K} \alpha_{1}\right.$ radiation, $\left.\lambda=1.5406 \AA\right)$ [31]. An Anton-Paar DHS 1100 chamber was used to heat the sample from room temperature up to $100^{\circ} \mathrm{C}$ (in steps of $2^{\circ} \mathrm{C}$ for temperatures close to the SPT, $5^{\circ} \mathrm{C}$ otherwise). XRD data have been analyzed with the DxTools program [33]. $\theta-2 \theta$ scans, recorded around the 002 reflection of $\mathrm{TiO}_{2}$, and RSMs recorded at $100^{\circ} \mathrm{C}$ around the 202 reflection of $\mathrm{TiO}_{2}$ are provided in the Supplementary Material (Fig. S1, S2 and S3). These measurements reveal that the $\mathrm{VO}_{2}$ films have a (001) orientation with the following epitaxial relationships: $(001)_{\mathrm{VO} 2} \|(001)_{\mathrm{TiO} 2}$ and $[100]_{V O 2} \|[100]_{\mathrm{TiO} 2}$ (for simplicity we use the $h k l$ indices of the high-temperature rutile phase of $\mathrm{VO}_{2}$ ). The volume fraction of the $\mathrm{M} 1$ phase has been determined from the temperature-resolved $\theta-2 \theta$ scans for all films following the approach detailed in [31] and recalled in the Supplementary Material. The transition temperatures upon heating and cooling are given by the peak positions in the first derivative of the $\log$ (resistivity) vs. temperature (respectively, volume fraction vs. temperature) curves. The corresponding MIT (respectively, SPT) temperature is obtained from the average between the heating and cooling transition temperatures.

(a)

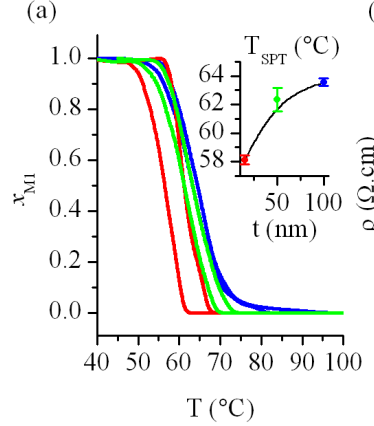

(b)

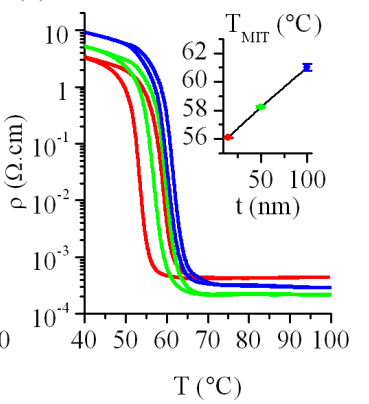

FIG. 1. (a) evolution of the volume fraction of the M1 phase vs. temperature, for a $15 \mathrm{~nm}$ (red), $50 \mathrm{~nm}$ (green) and 100 nm thick film (blue). Inset: evolution of the SPT temperature with thickness. (b) evolution of the resistivity vs. temperature, for a $15 \mathrm{~nm}$ (red), $50 \mathrm{~nm}$ (green) and $100 \mathrm{~nm}$ thick film (blue). Inset: evolution of the MIT temperature with thickness.

The evolution with temperature of the volume fraction of the M1 phase, for the three different film thicknesses, is displayed in Fig.1 (a) where the SPT hysteresis is clearly observed. For decreasing film thickness, the
SPT is shifted towards lower temperatures and spreads over a broader range of temperatures. Although the quantitative evolution of the SPT in only scarcely addressed in $\mathrm{VO}_{2}$ studies, this evolution is similar to the one observed for the MIT in the $\mathrm{VO}_{2} / \mathrm{TiO}_{2}(001)$ system $[2,5,9,12,14]$. This is further confirmed by our electrical measurements (Fig.1 (b)) where the MIT is indeed broadened and shifted towards lower temperature for decreasing film thickness. In the insets of Fig.1 (a) and (b), the transition temperatures are plotted as a function of the film thickness. It can be noted that there is a difference of $\sim 2^{\circ} \mathrm{C}$ between the MIT and the SPT; since the electrical and XRD measurements have been carried out with two different heating devices, the observed difference is certainly not relevant since absolute errors in temperature readings can not be definitely ruled out. However, the evolution of both transitions is similar, with a shift of the SPT and the MIT towards lower temperature with decreasing thickness. Such a behavior is in general attributed to a compression of the $c_{R}$ axis induced by tensile interfacial strain, the amount of which increases with decreasing thickness $[2,5,9,12,14]$. We investigate this interpretation below. It also can be noticed that the MIT temperature of the $15 \mathrm{~nm}$ thick film $\left(56^{\circ} \mathrm{C}\right)$ differs from previously published values for $\mathrm{VO}_{2} / \mathrm{TiO}_{2}(001)$ films with a similar thickness, $20^{\circ} \mathrm{C}[10,12], 43^{\circ} \mathrm{C}$ [9] and $44^{\circ} \mathrm{C}$ [15]. Moreover, when decreasing the film thickness from 100 to $15 \mathrm{~nm}$ the resistivity ratio between the insulating and conducting states devreases from $6.4 \times 10^{4}$ to $1.4 \times 10^{4}$ which suggest the presence of an interfacial region with degraded electrical properties. These two last observations are further discussed below.

The coefficient of thermal expansion along the $a$ axis of $\mathrm{TiO}_{2}$ and $\mathrm{VO}_{2}(\mathrm{R})$ have been determined by Rao et al. $[34,35]$ :

$$
\begin{array}{r}
\alpha_{a}^{T i O 2}=7.249 \times 10^{-6}+2.198 \times 10^{-9}(T-273) \\
+1.298 \times 10^{-12}(T-273)^{2} \\
\alpha_{a}^{V O 2}=5.828 \times 10^{-6}-7.091 \times 10^{-9}(T-273) \\
+6.946 \times 10^{-12}(T-273)^{2}
\end{array}
$$

where $T$ is the temperature (in Kelvins). With these values, the misfit strain $\left(\mathrm{a}_{\mathrm{TiO} 2}-\mathrm{a}_{\mathrm{VO}}\right) / \mathrm{a}_{\mathrm{VO} 2}$ at the growth temperature $\left(500^{\circ} \mathrm{C}\right)$ is $1.04 \%$ (tensile). Such high strain values usually give rise to strain relaxation via the formation of misfit dislocations at relatively low film thicknesses [36]. Among the possible slip systems in the rutile structure [37], only the $1 / 2<101>\{10 \overline{1}\}$ system has a non-zero edge component of the Burgers vector in the (001) plane and is hence able to relax interfacial strain. With this slip system, the Matthews and Blakeslee criterion [36, 38] yields a critical thickness $t_{c}=3.9 \mathrm{~nm}$ above which dislocations glide to the interface in order to progressively relieve the elastic strain energy. Progressive strain relaxation between the $15 \mathrm{~nm}$ 
and $100 \mathrm{~nm}$ thick film might hence explain the behavior of the MIT and the SPT.

In order to evaluate the state of strain within the $\mathrm{VO}_{2}$ films, RSMs were recorded around the 202 reflexions of $\mathrm{VO}_{2}$ and $\mathrm{TiO}_{2}$ (Supplementay Material, Fig. S3). The measurements were carried out at $100^{\circ} \mathrm{C}$ so as to stabilize the $\mathrm{VO}_{2}(\mathrm{R})$ phase, hence avoiding transformation strains and twins that might occur during cooling down through the SPT. The in-plane $\left(a_{V O 2}\right)$ and out-of-plane $\left(c_{\mathrm{VO} 2}\right)$ lattice parameters of the film were obtained from the coordinates of the 202 reflection of $\mathrm{VO}_{2}$ in the reciprocal space: $a_{V O 2}=4 \pi / Q_{x}^{202}$ and $c_{V O 2}=4 \pi / Q_{z}^{202}$. The evolution of $a_{V O 2}$ and $c_{V O 2}$ are displayed in Fig.2 (a) and are indeed consistent with a decrease of the in-plane tensile strain (and out-of-plane compressive strain) upon increasing film thickness. Using the atomic structure of rutile, the apical and equatorial $\mathrm{V}-\mathrm{O}$ distances can be respectively computed as 0.42426 $a_{V O 2}$ and $\left(c_{V O 2}^{2}+0.32 a_{V O 2}{ }^{2}\right)^{1 / 2} / 2$ [8]. The corresponding distance are plotted in Fig.2 (b). It can be observed that, whereas the equatorial distance remains roughly constant (decreasing from 1.924 to $1.922 \AA$ ), the apical distance decreases from 1.94 to $1.93 \AA$ which increases the p-d hybridization and up-shifts the $\pi^{*}$ level, thereby widening the band gap $[8,9]$, hence the observed increase of $\mathrm{T}_{M I T / S P T}$ for increasing thickness.
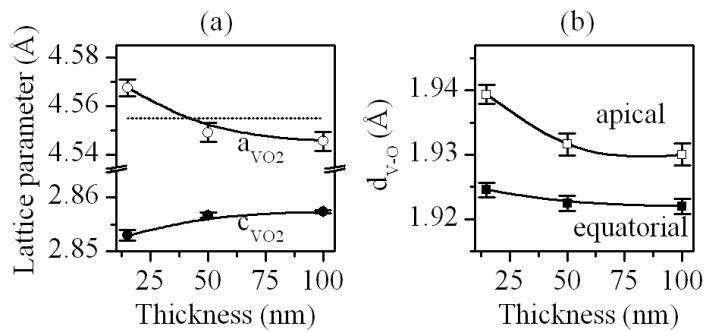

FIG. 2. (a) evolution of $a_{\mathrm{VO} 2}$ (open circles) and $c_{\mathrm{VO} 2}$ (filled circles) deduced from the RSMs as a function of thickness. The dotted line is the bulk value of $a_{V O 2}$ published by McWhan et al. [39]. (b) evolution of the apical (open squares) and equatorial (filled squares) $\mathrm{V}-\mathrm{O}$ distances.

Although this interpretation fits well within the above molecular orbital scheme, a closer examination of the in-plane state of strain seems inconsistent with this picture. Evaluating the actual state of strain of $\mathrm{VO}_{2}(\mathrm{R})$ is not straightforward, in part because this requires a precise knowledge of the strain-free bulk lattice parameters $\left(a_{b}, c_{b}\right)$. Commonly used value are those provided by McWhan et al. [39]. The corresponding value of $a_{b}$ is plotted in Fig.2 (a) (dotted line). It can be seen that between the $15 \mathrm{~nm}$ and the $50 \mathrm{~nm}$ thick films, the inplane strain changes from tensile $\left(a_{V O 2}>a_{b}\right.$, as expected from the lattice mismatch) to compressive $\left(a_{V O 2}<a_{b}\right)$. However, several others values of $a_{b}$ can be found in the literature with fluctuations as high as $0.04 \AA$, which obviously prohibits any possible quantitative interpretation based on these values. The most likely explanations for this dispersion of the data is that $\mathrm{VO}_{2}(\mathrm{R})$ is not stable at room temperature, so that the observed dispersion merely stems from the various means used to stabilize this phase. Table I summarizes the values for which the synthesis and measurement conditions (in particular the temperature) where precisely documented [39-42].

\begin{tabular}{ccccc}
\hline $\mathrm{a}_{b}(\AA)$ & $\mathrm{c}_{b}(\AA)$ & $k=\frac{c_{b}}{a_{b}}$ & $\mathrm{~T}\left({ }^{\circ} \mathrm{C}\right)$ & Reference \\
\hline 4.55349 & 2.84944 & 0.62577 & 65.6 & {$[40]$} \\
4.554 & 2.856 & 0.62714 & 127 & {$[41]$} \\
4.5546 & 2.8514 & 0.62605 & 87 & {$[39]$} \\
4.5496 & 2.8542 & 0.62735 & 87 & {$[42]$} \\
\hline
\end{tabular}

TABLE I. Published lattice parameters of $\mathrm{VO}_{2}(\mathrm{R})$. The measurement temperatures and the references are given in the last two columns.

The state of strain must therefore be computed without any explicit reference to $a_{b}$. Assuming that the geometry of the unit-cell is known (i.e. the $c_{b} / a_{b}$ ratio, noted $k$ ), the actual strain-free lattice parameter of $\mathrm{VO}_{2}(\mathrm{R})$ can be deduced from the values $a_{\mathrm{VO} 2}$ and $c_{\mathrm{VO} 2}$ determined above $[43,44]$ :

$$
a_{b}=\frac{(1-\nu) c_{V O 2}+2 \nu k a_{V O 2}}{(1+\nu) k}
$$

where $\nu$ is the Poisson's ratio which has been experimentally determined as $\nu=0.249$ [8]. With this value, the in-plane strain is $e_{x x}=\left(a_{V O 2}-a_{b}\right) / a_{b}$. The evolution of $e_{x x}$ as a function of the film thickness is plotted in Fig.3 (a). The grey area and the dotted lines indicate the range of values spanned by $e_{x x}$ using the different $k$ values reported in Table I. The full lines correspond to the average value. All values correspond to a temperature of $100^{\circ} \mathrm{C}$. Two important conclusions can be drawn:

(i) The $15 \mathrm{~nm}$ thick film experiences tensile strain, but strain relaxation already takes place since the observed value $(0.2 \%)$ is significantly lower than the misfit strain $\left(0.92 \%\right.$ as computed at $\left.100^{\circ} \mathrm{C}\right)$ which corresponds to a $\sim 78 \%$ relaxation rate. This feature certainly explains the difference with previous reports of the MIT occuring close to room temperature since the corresponding films were almost pseudomorphically strained [10, 12]. Moreover, the presence of relaxation-induced misfit dislocations might also explain the degradation of the resistivity ratio with decreasing film thickness as observed in previous studies $[9,31]$.

(ii) The $50 \mathrm{~nm}$ and $100 \mathrm{~nm}$-thick film experience compressive strain $(-0.12 \%$ and $-0.18 \%$, respectively). It can also be observed that these conclusions remain valid whatever the value of $k$ is used in the calculation. The only possible explanation for the compressive state of strain is the film/substrate thermal expansion mismatch, which yields a strain $e_{t h}(T)=\int_{T}^{T g}\left[\alpha_{V O 2}(T)-\alpha_{T i O 2}(T)\right] d T$, where $T_{g}$ is the growth temperature and $T$ the measurement temperature $\left(100^{\circ} \mathrm{C}\right)$. Using Eq. 1 and Eq. 2, this gives $e_{t h}=-0.14 \%$ (Fig.3 (a)). For the thickest film (for which the relaxation of the misfit strain can be reasonably assumed to be complete, so that the only remaining source of strain is of thermal origin) the value of thermal strain clearly falls in 

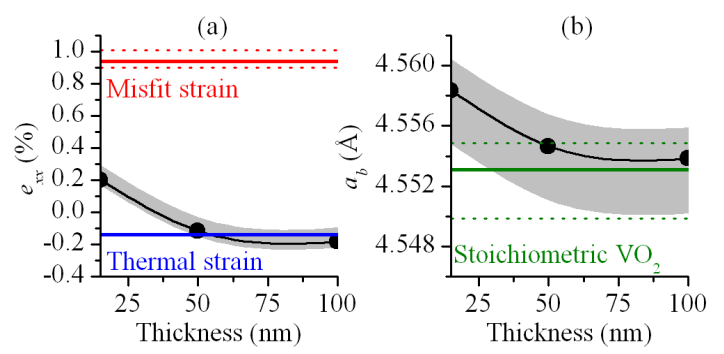

FIG. 3. (a) evolution of the in-plane strain (black line and circles) at $100^{\circ} \mathrm{C}$, deduced from the RSMs with increasing film thickness. The gray area correspond to the possible values obtained with different $c_{b} / a_{b}$ ratios. The red line correspond to the misifit strain at $100^{\circ} \mathrm{C}$. The dotted line correspond to the possible values obtained with different $c_{b} / a_{b}$ ratios. The blue line is the theoretical thermal strain. (b) evolution of the bulk (strain-free) lattice parameter deduced from the RSMs with increasing film thickness. The gray area has the same meaning as in (a). The green line is the average of the bulk values given in Table I. The dotted lines correspond to the maximum deviation from this average.

the range of possible strain values, which unambiguously confirms the role of the thermal expansion mismatch.

The observation of in-plane compressive strain is in disagreement with the values of both the MIT and the SPT temperatures $\left(<68^{\circ} \mathrm{C}\right)$ which imply a tensile state of strain for all films (with a larger level for the thinnest films). This discrepancy can be rationalized by examining the evolution of the strain-free lattice parameter, as determined from Eq. 3. The evolution with thickness is plotted in Fig.3 (b). It is striking to notice that, for all films considered, $a_{b}$ is larger than the expected value (for the average as well as for the extreme values), and decreases with increasing film thickness. This demonstrates the existence of an interfacial region with increased lattice parameters ; the fact that the strain-free lattice parameter decreases with increasing film thickness is a consequence of the decreasing weight of the interfacial region to the overall XRD measurement when the film thickness increases. Previous studies revealed the existence of a $1.7 \mathrm{~nm}$-thick interfacial region where $\mathrm{Ti}$ diffused from the substrate into the $\mathrm{VO}_{2}$ film [12]. Since the ionic radius of $\mathrm{Ti}^{4+}$ in a 6 -fold coordinate environment is $0.605 \AA$ (vs. $0.58 \AA$ for $\mathrm{V}^{4+}$ ), $\mathrm{Ti}$ diffusion might explain the swelling of the unit-cell as well as the degradation of the electrical properties [12] observed in Fig. 1 (b). However, contrarily to what is observed experimentally (especially for the thickest film), Ti diffusion should stabilize the monoclinic phase and hence shift the MIT towards higher temperatures [12] (i.e. $>68^{\circ} \mathrm{C}$ ). Another hypothesis is the presence of oxygen vacancies located at the interface. This mechanism is facilitated by the multiple oxidation state of the $\mathrm{V}$ ions and has been observed in other metal oxide compounds like $\mathrm{SmNiO}_{3}[43,45]$ as a means to relax epitaxial strain. The formation of oxygen vacancies yields a swelling of the unit-cell, as a consequence of both the formation of $\mathrm{V}^{3+}$ ions with a larger ionic radius than $\mathrm{V}^{4+}$, which are required by charge neutrality, and unscreened electrostatic repulsion between neighboring $\mathrm{O}^{2-}$ ions $[19,30,31]$. The net result is a lowering of the misfit strain at the growth temperature. Although Ti diffusion can not be firmly ruled out without further characterization, the existence of $\mathrm{O}$ vacancies might explain the stabilization of the rutile phase, the swelling of the unit-cell phase and, in the present case, this effect would be significant enough to counterbalance the effect of in-plane compressive strain. This is similar to what was observed in $\mathrm{VO}_{2}$ films grown of $\mathrm{MgF}_{2}(110)$ substrates, where oxygen vacancies were intentionally introduced during growth [29]. Besides, the presence of misfit dislocations explains the lowering of the resistivity ratio for the thinner films. Further experiments, using X-ray photoelectron spectroscopy and transmission electron microscopy, are required to confirm the presence of $\mathrm{O}$ vacancies (and/or Ti diffusion) as well as to precisely characterize the misfit dislocations.

Summarizing, using XRD we have shown that the evolution of the MIT and the SPT of $\mathrm{VO}_{2}$ films, of increasing thickness, grown on $\mathrm{TiO}_{2}(001)$ substrates is governed by the competing effects of lattice mismatch, thermal expansion mismatch and composition. Whereas lattice mismatch promotes in-plane tensile strain, hence stabilizing the rutile phase, the thermal expansion mismatch combined with strain relaxation progressively changes the sign of the in-plane strain. Nonetheless the effect of compressive in-plane strain on the MIT and the SPT is counterbalanced by a swelling of the unit-cell at the interface. These results highlight the fact that for $\mathrm{VO}_{2}$ studies, and very likely for other transition metal oxides as well, the sole epitaxial strain is not sufficient to explain the electrical properties of the materials. The role of thermal expansion mismatch and and composition variations (either non-stoichiometry or film/substrate interdiffusion) must be taken into account.

\section{Supplementary Material}

The Supplementary Material contains wide-range $\theta-$ $2 \theta$ scans recorded at room temperature, temperatureresolved $\theta-2 \theta$ around the 002 reflection of $\mathrm{VO}_{2}$ and $\mathrm{TiO}_{2}$, and RSMs recorded at $100^{\circ} \mathrm{C}$ around the 202 reflection of $\mathrm{VO}_{2}$ and $\mathrm{TiO}_{2}$. Details regarding the determination of phase volume fractions from the simulation of XRD data are also provided.

\section{Acknowledgment}

VT is grateful to région Limousin (France) and Labex SigmaLim for financial support. 
[1] J. Pouget, H. Launois, J. D'Haenens, P. Merenda, and T. Rice, Phys. Rev. Lett. 35, 873 (1975).

[2] Y. Muraoka and Z. Hiroi, Appl. Phys. Lett. 80, 583 (2002).

[3] K. Nagashima, T. Yanagida, H. Tanaka, and T. Kawai, Phys. Rev. B 74, 172106 (2006).

[4] B. Lazavorits, K. Kim, K. Haule, and G. Kotliar, Phys. Rev. B 81, 115117 (2010).

[5] Y. Gu, J. Cao, J. Wu, and L.-Q. Chan, J. Appl. Phys. 108, 083517 (2010).

[6] T. Kikuzuki and M. Lippmaa, Appl. Phys. Lett. 96, 132107 (2010).

[7] E. Abreu, M. Liu, J. Lu, K. G. West, S. Kittiwatanakul, W. Yin, S. A. Wolf, and R. D. Averitt, New J. Phys. 14, 083026 (2012).

[8] N. Aetukuri, A. Gray, M. Drouard, M. Cossale, L. Gao, A. Reid, R. Kukreja, H. Ohlag, C. Jenkins, E. Arenholz, K. Roche, H. Durr, M. Samant, and S. Parkin, Nature Phys. 10, 1038 (2013).

[9] L. Fan, S. Chan, Z. Luo, Q. Liu, Y. Wu, L. Song, D. Ji, P. Wang, W. Chu, C. Gao, C. Zou, and Z. Wu, Nano Lett. 14, 4036 (2014).

[10] T. Yajima, Y. Ninomiya, T. Nishimura, and A. Toriumi, Phys. Rev. B 91, 205102 (2015).

[11] H. Qiu, M. Yang, Y. Dong, H. Xu, B. Hong, Y. Gu, Y. Yang, C. Zou, Z. Luo, and C. Gao, New J. Phys. 17, 113016 (2015).

[12] H. Paik, J. A. Moyer, T. Spila, J. W. Tashman, J. A. Mundy, E. Freeman, N. Shukla, J. M. Lapano, R. EngelHerbert, W. Zander, J. Schubert, D. Muller, S. Datta, P. Schiffer, and D. G. Schlom, Appl. Phys. Lett. 107, 163101 (2015).

[13] L. Bai, Q. Li, S. A. Corr, Y. Meng, C. Park, S. V. Sinogeikin, C. Ko, J. Wu, and G. Shen, Phys. Rev. B 91, 104110 (2015).

[14] N. F. Quackenbush, H. Paik, M. J. Wahila, S. Sallis, M. E. Holtz, X. Huang, A. Ganose, B. J. Morgan, D. O. Scanlon, Y. Gu, F. Xue, L.-Q. Chen, G. E. Sterbinsky, C. Schlueter, T.-L. Lee, J. C. Woicik, J.-H. Guo, J. D. Brock, D. A. Muller, D. A. Arena, D. G. Schlom, and L. F. J. Piper, Phys. Rev. B 94, 085105 (2016).

[15] M. Yang, Y. Yang, B. Hong, L. Wang, K. Hu, Y. Dong, H. Xu, H. Huang, J. Zhao, H. Chen, L. Song, H. Ju, J. Zhu, J. Bao, X. Li, Y. Gu, T. Yang, X. Gao, Z. Luo, and C. Gao, Sci. Rep. 6, 23119 (2016).

[16] Y. Chen, S. Zhang, F. Ke, C. Ko, S. Lee, K. Liu, B. Chen, J. W. Ager, R. Jeanloz, V. Eyert, and J. Wu, Nano Lett. 17, 2512 (2017).

[17] J. Goodenough, J. Solid State Chem. 3, 490 (1971).

[18] V. Eyert, Ann. Phys. 11, 650 (2002).

[19] C. H. Griffiths and H. K. Eastwood, J. Appl. Phys. 45, 2201 (1974).

[20] W. Brückner, W. Moldenhauer, H. Wich, E. Wolf, H. Oppermann, U. Gerlach, and W. Reichelt, Phys. Stat. Sol. (a) 29, 63 (1975).

[21] S. Zhang, I. S. Kim, and L. J. Lauhon, Nano Lett. 11, 1443 (2011).

[22] L. Fan, Y. Wu, C. Si, G. Pan, C. Zou, and Z. Wu, Appl. Phys. Lett. 102, 011604 (2013).

[23] L. Yeo, A. Srivastava, M. Majidi, R. Sutarto, F. HE, S. Poh, C. Diao, X. Yu, M. Motapothula, S. Saha, S. Ojha, D. Kandjilal, P. Trevisanutto, M. Breese,
T. Venkatesan, and A. Rusydi, Phys. Rev. B 91, 081112 (2015).

[24] J. Jeong, N. Aetukuri, D. Passarello, S. Conradson, M. Samant, and S. Parkin, PNAS 112, 1013 (2015).

[25] H.-T. Zhang, L. Zhang, D. Mukherjee, Y.-X. Zheng, R. C. Haislmaier, N. Alem, and R. Engel-Herbert, Nature Comm. 6, 8475 (2015).

[26] H. T. Zhang, L. Guo, G. Stone, L. Zhang, Y. X. Zheng, E. Freeman, D. W. Keefer, S. Chaudhuri, H. Paik, J. A. Moyer, M. Barth, D. G. Schlom, J. V. Badding, S. Datta, V. Gopalan, and R. Engel-Herbert, Adv. Funct. Mater. 26, 6612 (2016).

[27] S. Chen, X. j. Wang, L. Fan, G. Liao, Y. Chen, W. Chu, L. Song, J. Jiang, and C. Zou, Adv. Funct. Mater. 26, 3532 (2016).

[28] Z. Zhang, F. Zuo, C. Wan, A. Dutta, J. Kim, J. Rensberg, R. Nawrodt, H. H. Park, T. J. Larrabee, X. Guan, Y. Zhou, S. M. Prokes, C. Ronning, V. M. Shalaev, A. Boltasseva, M. A. Kats, and S. Ramanathan, Phys. Rev. Applied 7, 034008 (2017).

[29] L. L. Fan, S. Chen, G. M. Liao, Y. L. Chen, H. Ren, and C. W. Zou, J. Phys.: Condens. Matter 28, 255002 (2016).

[30] L. Chen, Y. Cui, S. Shi, B. Liu, H. Luo, and Y. Gao, RSC Adv. 6, 86872 (2016).

[31] V. Théry, A. Boulle, A. Crunteanu, J. C. Orlianges, A. Beaumont, R. Mayet, A. Mennai, F. Cosset, A. Bessaudou, and M. Fabert, Phys. Rev. B 93, 184106 (2016).

[32] V. Théry, A. Boulle, A. Crunteanu, J. C. Orlianges, A. Beaumont, R. Mayet, A. Mennai, F. Cosset, A. Bessaudou, and M. Fabert, J. Appl. Phys. 121, 055303 (2017).

[33] A. Boulle, J. Appl. Crystallogr. 50, 967 (2017).

[34] K. V. K. Rao, S. V. N. Naidu, and L. Iyengar, J. Phys. Soc. Jpn. 23, 1380 (1967).

[35] K. V. K. Rao, S. V. N. Naidu, and L. Iyengar, J. Am. Ceram. Soc. 53, 124 (1970).

[36] S. C. Jain, A. H. Harker, and R. A. Cowley, Philos. Mag. A 75, 1461 (1997).

[37] K. H. G. Ashbee, R. E. Smallman, and G. K. Williamson, Proc. R. Soc. A 276, 542 (1963).

[38] J. Matthews and A. Blakeslee, J. Cryst. Growth 27, 118 (1974).

[39] D. McWhan, M. Marezio, J. Remeika, and P. Dernier, Phys. Rev. B 10, 490 (1974).

[40] D. Kucharczyk and T. Niklewski, J. Appl. Crystallogr. 12, 370 (1979).

[41] K. D. Rogers, Powder Diffr. 8, 240 (1993).

[42] M. Marezio, B. McWhan, J. Remeika, and P. Dernier, Phys. Rev. B 5, 2541 (1972).

[43] F. Conchon, A. Boulle, R. Guinebretière, C. Girardot, S. Pignard, J. Kreisel, F. Weiss, E. Dooryhée, and J.-L. Hodeau, Appl. Phys. Lett. 91, 192110 (2007).

[44] A. Boulle, S. Kilburger, P. D. Bin, E. Millon, C. D. Bin, R. Guinebretière, and A. Bessaudou, J. Phys. D: Appl. Phys. 42, 145403 (2009).

[45] F. Conchon, A. Boulle, R. Guinebretière, E. Dooryhée, J.-L. Hodeau, C. Girardot, S. Pignard, J. Kreisel, and F. Weiss, J. Phys.: Condens. Matter 20, 145216 (2008). 